Internal Report

DESY M 02-02

December 2002

\title{
Review of Experimental Results on High-Brightness Photo-Emission Electron Sources
}

Ph. Piot

Deutsches Elektronen-Synchrotron DESY, Hamburg 
DESY behält sich alle Rechte für den Fall der Schutzrechtserteilung und für die wirtschaftliche Verwertung der in diesem Bericht enthaltenen Informationen vor.

DESY reserves all rights for commercial use of information included in this report, especially in case of filing application for or grant of patents.

"Die Verantwortung für den Inhalt dieses

Internen Berichtes liegt ausschließlich beim Verfasser" 


\title{
REVIEW OF EXPERIMENTAL RESULTS ON HIGH-BRIGHTNESS PHOTO-EMISSION ELECTRON SOURCES
}

\author{
PH. PIOT \\ Deutsches Elektronen-Synchrotron (DESY), D-22603 Hamburg, Germany. \\ E-mail: Philippe.piot@desy.de
}

\begin{abstract}
The generation of electron beams with high phase space density is one of the principal challenges for the next generation of ultra-short wavelength light sources and for the foreseen linear colliders projects. In this paper we review some of the recent experimental advances in the field of electron sources based on the photoelectric effect.
\end{abstract}

\section{Introduction}

\subsection{Overview}

In the recent years electron sources based on the photoelectric effect have supported operation of many accelerators ranging from colliders (e.g. SLC) to novel light sources (e.g. at ANL, BNL, DESY, JLAB). This kind of electron sources have been supporting advanced beam physics experiments such as plasma-driven accelerator (e.g. ANL, FNAL) and Thomson scattering (E.G. LLNL).

The photo-emission electron source (see earlier reviews from Sheffield ${ }^{1}$ and O'Shea ${ }^{2}$ ) offers many advantages compared to the other popular technologies (e.g. thermionic emission). Firstly, there is a good handle on the initial conditions of the emitted electron bunch. Such initial conditions (transverse and longitudinal distribution) is controlled via the photocathode drive-laser parameters (pulse length, spot size on the cathode, intensity, etc...) and turn out to be an important feature for minimizing the transverse emittance growth. With the availability of ultrashort pulse lasers, the electron bunch length can be made small compared to the radio-frequency ( $\mathrm{rf}$ ) cavity wavelength thereby removing the need of sub-harmonic bunching schemes as in injectors based on thermionic sources. On the other hand, the charge density that can generally be reached with photocathodes is of the $\mathrm{kA} . \mathrm{cm}^{-2}$ level, approximately two orders of magnitude above thermionic emitters. Finally the use of a polarized drive-laser enables the production of polarized photoelectrons in virtue of the angular momentum conservation.

Because of the charge density considered, the photo-electrons need to be quickly accelerated in order to significantly decrease the space charge forces within the bunch. Hence the photocathode is located on the back-plate of the half-cell of a radio-frequency cavity (rf-gun) or at an extremity of a high-voltage gap (dc-gun) as schematized in Figure 1.

An electron source is generally integrated within an "injector" consisting of accelerating section(s) and focusing element(s) configurated in such a way to take

piot gunrev: submitted to World Scientific on October 23, 2002 


\begin{tabular}{|c|c|c|c|}
\hline Facility, Location & $\begin{array}{l}\text { Gun Type } \\
f(\mathrm{GHz})\end{array}$ & \# of cell & Mission \\
\hline IRDemo, JLab ${ }^{6}$ & $\mathrm{dc}$ & - & high average power FEL \\
\hline $\mathrm{ATF}, \mathrm{BNL}^{7}$ & $\mathrm{rf}, 2.86$ & 1.6 & multi-disciplinary facility \\
\hline LEUTL, ANL ${ }^{8}$ & rf, 2.86 & 1.6 & UV SASE-FEL \\
\hline SDL, BNL 9 & rf, 2.86 & 1.6 & UV SASE-FEL \\
\hline CTF, CERN 10 & $\mathrm{rf}, 3.0$ & 1.5 & CLIC test facility \\
\hline AFEL, LANL 11 & $\mathrm{rf}, 1.3$ & 10.5 & IR SASE-FEL \\
\hline AWA, ANL ${ }^{12}$ & $\mathrm{rf}, 1.3$ & 1.6 & wakefield acceleration \\
\hline GTF, SLAC ${ }^{13}$ & rf, 2.86 & 1.6 & LCLS test injector \\
\hline TTF-1/2, DESY-HH ${ }^{14}$ & $\mathrm{rf}, 1.3$ & 1.6 & $\begin{array}{l}\text { TESLA test facility } \\
+ \text { VUV SASE-FEL }\end{array}$ \\
\hline PITZ, DESY-Z ${ }^{15}$ & rf, 1.3 & 1.6 & injector test facility \\
\hline FNPL, FNAL ${ }^{16}$ & $\mathrm{rf}, 1.3$ & 1.6 & multi-disciplinary facility \\
\hline $\mathrm{MIT}^{17}$ & $\mathrm{rf}, 17.14$ & 1.5 & multi-disciplinary facility \\
\hline Tokai 18 & $\mathrm{rf}, 2.86$ & 1.6 & multi-disciplinary facility \\
\hline NEPTUNE, UCLA 19 & rf, 2.86 & 1.6 & multi-disciplinary facility \\
\hline PEGASSUS, UCLA 20 & rf, 2.86 & 10.5 & multi-disciplinary facility \\
\hline APLE, BOEING ${ }^{21}$ & rf, 0.433 & 1.5 & high average power FEL \\
\hline ELSA, CEA ${ }^{22}$ & rf, 0.144 & 1.5 & $\begin{array}{l}\text { high average power FEL } \\
+ \text { Thomson X-ray source }\end{array}$ \\
\hline DROSSEL, FZR ${ }^{23}$ & srf, 1.3 & 0.5 & supercond. gun test facility \\
\hline
\end{tabular}

Table 1. List of some of the representative accelerator test facilities based on photo-electron sources.

advantage of the emittance compensation mechanism ${ }^{3,4,5}$. Some possible configurations are presented in Figure 2. For the rf-gun set-up, the two variants widely used are the so-called integrated and split injectors ${ }^{24}$. The former configuration (e.g. AFEL at LANL) uses long multi-cell rf-gun cavity with low peak electric field whereas the latter one (e.g. ATF at BNL) is based on a 1-1/2-cell rf-gun cavity operating with high peak electric field closely followed by a booster linac.

\subsection{Important parameters}

The figure-of-merit when considering the application of the electron source to drive free-electron lasers (FEL) or in the framework of linear colliders is the phase space density. Given the normalized transverse and longitudinal rms emittances $\tilde{\varepsilon}_{x, y}$ and $\tilde{\varepsilon}_{z}$ respectively and the bunch charge $Q$, we define the brightness as the ratio of the bunch charge to the hyper-volume it occupies in the 6-D phase space:

$$
\mathcal{B} \doteq m_{e} c^{2} \frac{Q}{\tilde{\varepsilon}_{z} \tilde{\varepsilon}_{x} \tilde{\varepsilon}_{y}} .
$$


a)

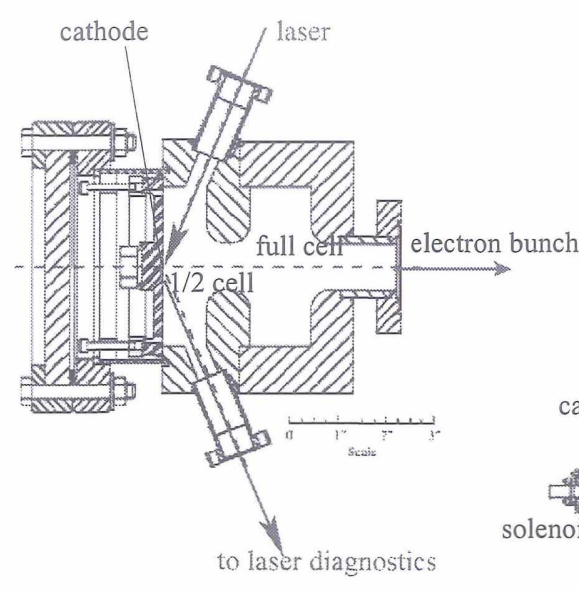

b)



Figure 1. Schematic rendition of an rf-gun (a) and dc-gun (b) based electron sources.



Figure 2. The various photo-emission source configuration used: rf-gun split (a) and integrated (b) design, and the dc-gun design (c).

Here $m_{e} c^{2}$ is the electron rest energy. In Eq. (1) the emittances are defined following:

$$
\tilde{\varepsilon}_{x}=\frac{1}{\left\langle p_{z}\right\rangle} \sqrt{\left\langle(x-\langle x\rangle)^{2}\right\rangle\left\langle\left(p_{x}-\left\langle p_{x}\right\rangle\right)^{2}\right\rangle-\left\langle(x-\langle x\rangle)\left(p_{x}-\left\langle p_{x}\right\rangle\right)\right\rangle^{2}}
$$


where $x$ and $p_{x}$ are the position and canonical momentum in the phase space. The same type of definition applies for the $y$ and $z$ emittances. In our definition the longitudinal emittance units are keV-mm. Eq. (1) can also be extended to the multibunch case. In the definition of such multibunch brightness, the charge and emittances are those computed over the considered train of bunch:

$$
\mathcal{B}_{M} \doteq m_{e} c^{2} \frac{Q_{M}}{\tilde{\varepsilon}_{z, M} \tilde{\varepsilon}_{x, M} \tilde{\varepsilon}_{y, M}} \times \mathcal{D},
$$

where $\mathcal{D}$ is the duty cycle and the subscripts $M$ indicated the quantities are taken over the macrobunch. Sources of degradation of this multibunch brightness essentially come from long range wakefields and rf stability issues.

Though it should be underscored that for some applications, the figure-of-merits such as emittance or brightness need to be considered over longitudinal slices along the bunch. For instance the self-amplified stimulated emission (SASE) FEL properties depend upon beam parameters within slices whose lengths are of the order of the so-called "cooperation length", that is the slippage length between the radiation field and the electron for one gain length. For an X-ray FEL (1 Angstrom wavelength) these slices are typically $\sim 1 \mu \mathrm{m}$ long.

\section{Photocathodes and drive-lasers}

Given the quantum efficency $\eta$ of the photocathode, the drive-laser energy $\mathcal{E}$ and wavelength $\lambda$, the charge $Q$ of the photo-emitted electron bunch is:

$$
Q=\eta \times \frac{e \lambda}{h c} \mathcal{E}, \text { or } Q[\mathrm{nC}] \simeq \eta[\%] \times \frac{\lambda[\mathrm{nm}]}{124} \mathcal{E}[\mu \mathrm{J}] .
$$

The latter equation is valid for a single-photon photoemission process. The most commonly used photocathode materials along with a few of their properties are summarized in Table 2.

Metallic photocathodes can be used with ultra-violet light as they are stable and "blind" to photons having lower energies. However, due to metallic reflection and internal absorption, the quantum efficiency of such materials is rather low (typically $\left.\eta \sim 10^{-4}\right)$.

In semiconductors photocathodes, the photons must impart enough energy to the electrons in the valence band to reach the conduction band and have enough energy left to escape the photocathode into the surrounding vacuum. The energy difference between the bottom of the valence band and the vacuum level is called the electron affinity $E_{a}$ and the total energy required is $E_{a}+E_{g}$ where $E_{g}$ is the energy gap between the top of the valence and the bottom of the conduction bands. Alkali Antimonide and Telluride (which are of the positive electron affinity $\left(E_{a}>0\right)$ type) have been used to produced unpolarized highly charged beams with a duty cycle similar to what is required for the TESLA linear collider.

Negative electron affinity (NEA) photoemitters are p-type semiconductors whose surface is treated to "bend" the band structure. In extreme cases, the vacuum level is lower than the bottom of the conduction band. The principal advantages

piot gunrev: submitted to World Scientific on October 23, 2002 


\begin{tabular}{lrrrrr}
\hline \hline Material & typ. Q.E. & $\begin{array}{r}\lambda \\
(\mathrm{nm})\end{array}$ & $\begin{array}{r}\text { lifetime } \\
\text { (days) }\end{array}$ & $\begin{array}{r}\text { vacuum } \\
\text { req. }(\mathrm{T})\end{array}$ & in use at \\
\hline $\mathrm{Cu}$ & $2 \times 10^{-4}$ & 260 & months & $\sim 10^{-7}$ & ATF, SDL, Tokai, \\
$\mathrm{Mg}$ & $2 \times 10^{-3}$ & 260 & months & $\sim 10^{-7}$ & ATF \\
Alkali Antimonide & $5 \times 10^{-2}$ & 532 & days & $\sim 10^{-10}$ & BOEING \\
Alkali telluride & $1 \times 10^{-2}$ & 260 & months & $\sim 10^{-9}$ & FNPL, TTF, \\
NEA GaAs (Cs) & $5 \times 10^{-2}$ & 780 & days & $\sim 10^{-11}$ & IrDemo \\
\hline \hline
\end{tabular}

Table 2. Commonly used cathode material and some of their typical characteristic.

of NEA photocathode include a longer wavelength operation and a higher quantum efficiency compared to the other photocathode types. An example of a NEA photoemitter is Gallium Arsenide ( $\mathrm{GaAs}$ ). It has been used as a photocathode in dc-guns to produce polarized beams with high duty cycle ${ }^{25}$.

Although metallic photocathodes can stand relatively poor vacuum, their quantum efficency is at the $10^{-4}$ level. Hence the required laser energy for producing $1 \mathrm{nC}$ of charge is of the order of $5 \mathrm{~mJ}$. Driving a high average current accelerator, e.g. an energy-recovery linac-based light source, would require a $\sim 100 \mathrm{~W}$ average power drive-laser if a metallic cathode would be used. Developing such a laser presents important technological challenges and instead, the so-called "ignited feedback regenerative amplifier" concept was proposed. In this latter scheme a part of the FEL radiation serves to drive the photocathode once a conventional drive-laser has been used for the start-up ${ }^{27}$. This concept was recently demonstrated at the TESLA test facility (TTF) where the light produced by the vacuum ultraviolet (VUV) SASE-FEL (wavelength $108 \mathrm{~nm}$ ) was reflected on a downstream mirror back to the Cesium Telluride cathode to regenerate electron bunches ${ }^{28}$.

The photocathode material along with the laser used to produce the electrons sets the initial "thermal" emittance that is the emittance generated because of the excess in kinetic energy of the photo-emitted electrons. The thermal emittance sets a lower limit on the beam emittance that can ideally be reached once the accelerator setup has been optimized. Given the excess kinetic energy $K$ of the photo-emitted electrons, the thermal emittance $\tilde{\varepsilon}_{t h}$ scales linearly with the drive-laser transverse spot size $r$ on the cathode as ${ }^{30}$ :

$$
\tilde{\varepsilon}_{t h} \simeq \frac{r}{2} \sqrt{\frac{2 K}{3 m_{e} c^{2}}}
$$

The latter equation assumes (1) the laser spot to be a uniform distribution and (2) the electrons to be emitted isotropically with the average kinetic energy $K$. To date, detailed measurements of thermal emittance have been reported for NEA GaAs and Copper cathodes only.

For GaAs cathodes, the transverse emittance measurements were performed at the Illinois/CEBAF polarized DC electron source ${ }^{31}$. The emittance was measured for different drive-laser wavelengths and spot sizes on the cathode. The deduced 
thermal transverse energy at $\lambda=710 \mathrm{~nm}$ was $K=37 \mathrm{meV}$. Recently, a technique to measure the energy distribution of electrons as a function of the transverse and longitudinal energies has been developed and applied to study the distribution of the photoemitted electron from a cooled $(T=90 \mathrm{~K})$ GaAs cathode ${ }^{32}$. It was shown that the obtained population distribution has a sharp peak for transverse thermal energies of about $5 \mathrm{meV}$ for $\lambda=800 \mathrm{~nm}$. The comparison of the two aforementioned measurements supports that NEA photoemitters such as GaAs photoemit electrons that are in thermal equilibrium with the crystal lattice. Therefore by cooling this type of cathode, bunches with reduced transverse emittances may be obtained. However, the response time of such photoemitters is typically $>10 \mathrm{ps}^{29}$ and prohibits a precise tailoring of the initial time-distribution.

For Copper cathodes, a similar measurement was performed at the Source Development Laboratory (SDL) of BNL ${ }^{33}$. The emittance measurement was conducted using an S-band rf-gun. To avoid emittance dilution due to rf effects, the gun field and bunch charge were optimized to insure the emittance to be dominated by its thermal contribution. From this latter measurement, the thermal energy was estimated to be $K=430 \mathrm{meV}$ for the wavelength $\lambda=260 \mathrm{~nm}$, which is one factor of magnitude above the GaAs measurement at room temperature. In Reference ${ }^{33}$, the large value of the measured thermal energy (theory predicts $260 \mathrm{meV}$ ) was attributed to the non perfect uniformity of the cathode surface: the roughness of the cathode surface can locally enhance the electric field ${ }^{34}$ and thus impacts the bunch thermal emittance.

\section{DC guns}

Only one high-brightness photo-emission dc-gun was operated to drive an FEL, the Ir-Demo, at Jefferson Lab. The nominal dc voltage was $350 \mathrm{keV}$ (corresponding to an electric field of $\sim 6 \mathrm{MV} / \mathrm{m}$ on the cathode surface) and bunch charges up to $130 \mathrm{pC}$ have been extracted from the GaAs cathode during nominal operation ${ }^{35}$. The gun was coupled to a so-called quarter CEBAF-cryomodule composed of two CEBAF-type 5-cell accelerating cavities with average accelerating gradient of about $10 \mathrm{MV} / \mathrm{m}$. During the FEL operation ${ }^{36}$, the electron beam emittance measured at the undulator location (at $45 \mathrm{MeV}$ ) was $\sim 8 \pm 1 \mathrm{~mm}$-mrad for $60 \mathrm{pC}$. Noteworthy, and this is the reason for this gun design, was the sustained lasing at $1.7 \mathrm{~kW}$ average power thanks to a bunch repetition rate of $75 \mathrm{MHz} \mathrm{CW}$. At that time, the maximum gun voltage was limited by breakdown due to field emission. Present progress in treating the electrodes using ion implantation foresees the operation of similar dc guns with electric fields as high as $25 \mathrm{MV} / \mathrm{m}^{37}$. A team at JLab has also developed a laser, suited for GaAs cathodes, capable of operating with repetition frequency as high as $2.5 \mathrm{GHz}^{38}$ thereby providing the possibility to operate a linac downstream of the dc-gun in CW mode that is with a repetition rate equal to the rf frequency of the accelerator. Such a type of operation with a dc-gun is foreseen in the proposed energy recovering linac light source at Cornell ${ }^{39}$.

piot gunrev: submitted to World Scientific on October 23, 2002 


\begin{tabular}{lrr}
\hline \hline & value & units \\
\hline bunch charge & 0.1 & $\mathrm{nC}$ \\
laser pulse length (rms) & 1 & $\mathrm{ps}$ \\
peak cathode E-field & 200 & $\mathrm{MV} / \mathrm{m}$ \\
beam energy & 1.05 & $\mathrm{MeV}$ \\
\hline typical set of data: & & \\
bunch charge & 0.5 & $\mathrm{nC}$ \\
beam energy & 1.05 & $\mathrm{MeV}$ \\
energy spread (rms) & 1.5 & $\%$ \\
$\tilde{\varepsilon}_{\perp}(\mathrm{rms})$ & 1 & $\mathrm{~mm}-\mathrm{mrad}$ \\
bunch length (rms) & 1 & $\mathrm{ps}$ \\
\hline \hline
\end{tabular}

Table 3. Laser and rf parameters for the MIT rf-gun. A set of achieved beam parameters is also given.

\section{Radio-frequency guns}

Many groups operate test facilities that incorporate rf-gun photo-electron sources. The frequency of rf-guns presently under operation ranges from $f=0.144$ to $17 \mathrm{GHz}$. On the beam dynamics point-of-view if one could scales the rf cavity and photocathode drive-laser parameters such that the Vaslov equation is kept frequencyinvariant ${ }^{40,41}$, one would gain in operating at higher frequency since the brightness would scale as $\mathcal{B} \propto f^{-2}$. Indeed letting the Vaslov equation frequency-invariant requires the peak electric field to be scaled linearly with the frequency. In extreme cases this may result in problem such as rf-breakdown and field-induced heating. For instance simply scaling the presently achieved peak electric field $(140 \mathrm{MV} / \mathrm{m})$ for an S-band $(f=2.856 \mathrm{GHz})$ gun to an X-band $(f=17.136 \mathrm{GHz})$ would result in the unrealistic peak electric field value of $720 \mathrm{MV} / \mathrm{m}$ !

\section{$4.1 X$-band}

The Plasma Science and Fusion Center of MIT has worked toward the development of a high-frequency gun operating at $17.136 \mathrm{GHz}$. This work was motivated by the aforementioned favorable scaling of brightness with the frequency. The gun is a 1.5 cell accelerating structure and incorporates two side wall couplers (one on each cell). It also includes a solenoid for emittance compensation. The main parameters are gathered in Table 3. A multi-slit mask technique was used to measure the transverse emittance just downstream of the rf-gun. For $50 \mathrm{pC}$ charge, the transverse emittance was estimated to be about $1 \mathrm{~mm}$-mrad. To date the peak electric field achieved on the cathode is $200 \mathrm{MV} / \mathrm{m}$ and has been limited by rf-breakdown, resulting in a beam energy of $1 \mathrm{MeV}$ approximately. A new gun ( 2.4 cell) will soon be built in order to boost the beam energy to $2 \mathrm{MeV}$. It will then be coupled to a $25 \mathrm{MV} 17.136 \mathrm{GHz}$ traveling wave linac.

piot gunrev: submitted to World Scientific on October 23, 2002 


\subsection{S-band}

The so-called BNL/SLAC/UCLA/ gun operating at $2.856 \mathrm{GHz}$ is one of the most popular configurations. It is used at BNL (both at ATF and SDL), ANL (LEUTL), UCLA (Neptune), and in several Japanese Laboratories (e.g. at Tokai). Since its first version, the rf-gun has been gradually improved (symmetrization of the cavity, new solenoids, new laser port location,...).

At ATF, the gun has driven many experiments and has been use to demonstrate the saturation of the VISA SASE-FEL experiment. At this facility, the emittance has been measured to be $0.8 \mathrm{~mm}$-mrad for a charge of $0.5 \mathrm{nC}$. Because of the low emittance and to avoid saturation of the beam viewers a special high-betatron function optics was set up and the emittance was inferred by a fit of the beam envelope observed on four viewers. The emittance has been measured at $70 \mathrm{MeV}$ i.e. after acceleration through a $3 \mathrm{~m}$ SLAC-type traveling wave section and the measurement involved the minimization of transverse tails induced by dipole mode wake-fields along with beam-based alignment of the quadrupoles. Another study conducted at this facility aimed to investigate the transverse emittance dependence upon the transverse laser spot uniformity on the copper cathode. The laser uniformity is required to be very homogeneous transversely to insure within the bunch that the radial space charge field is linearly dependent on the radius (and thus does not yield a transverse emittance growth). Using neutral density matrix masks of different transmissions, the uniformity of the laser spot was varied and an increase of the emittance by a factor $>2$ was observed as the uniformity was changed from a contrast of 0 (uniform spot) to $50 \%{ }^{42}$.

At the source development laboratory (SDL) of BNL, an extended study on the DUV-FEL injector performance has been conducted. Two of the three accelerating sections that compose the accelerator have been operated at zero-crossing to impart a linear correlation between time and energy. The vertical emittance was then measured downstream of an horizontally bending spectrometer dipole. Therefore observing the vertical beam spot variation versus the strength of an upstream quadrupole for various energy (i.e. time) slice provides the slice emittance ${ }^{44}$. At $10 \mathrm{pC}$, slice emittances close the thermal emittance were measured ( $0.7 \mathrm{~mm}$-mrad over $200 \mathrm{fs}$ time slices).

Bunch compression using velocity bunching has also been attempted, and peak currents similar to those obtained by magnetic compression were obtained by running the first accelerating section far off-crest ${ }^{45}$.

At SLAC, the gun test facility (GTF) aims to optimize the LCLS injector and demonstrate the parameters required for the LCLS FEL project. Measurements of transverse and longitudinal emittance for various conditions have been conducted ${ }^{46}$. Noteworthy was the technique used to measure the longitudinal emittance: energy profiles downstream of the booster linac have been taken for various phase settings of the booster and a tomographic reconstruction technique in conjunction with particle tracking was used to infer the longitudinal phase space

piot gunrev: submitted to World Scientific on October 23, 2002 


\begin{tabular}{lrrrrrr}
\hline \hline & ATF & SDL & GTF & SHI & CLIC & units \\
\hline bunch charge & 1 & 1 & 1 & 1 & 100 & $\mathrm{nC}$ \\
laser pulse length (rms) & 3 & 2 & 1.3 & 4 & 4 & $\mathrm{ps}$ \\
peak cathode E-field & 120 & 100 & 120 & 100 & 105 & $\mathrm{MV} / \mathrm{m}$ \\
beam energy & 70 & 200 & 30 & 14 & 40 & $\mathrm{MeV}$ \\
\hline typical set of data: & & & & & & \\
bunch charge & 0.5 & 0.2 & 0.2 & 1 & 100 & $\mathrm{nC}$ \\
energy & 70 & 70 & 30 & 14 & 40 & $\mathrm{MeV}$ \\
energy spread (rms) & $0.03(\mathrm{u})$ & 0.05 & $0.025(\mathrm{u})$ & 0.25 & - & $\%$ \\
$\tilde{\varepsilon}_{\perp}(\mathrm{rms})$ & 0.8 & 1.5 & 1.5 & 1.2 & 100 & $\mathrm{~mm}-\mathrm{mrad}$ \\
bunch length (rms) & 3 & 2 & 1.8 & 4 & 4.3 & $\mathrm{ps}$ \\
\hline \hline
\end{tabular}

Table 4. Typical photocathode drive-laser and rf parameters for various S-band guns. A set of achieved beam parameters is also given. All the gun presented in this Table operate at $f=2.856 \mathrm{GHz}$ apart from the CLIC gun for which $f=3.0 \mathrm{GHz}$. Note: "(u)" in the "energy spread" line indicates the quoted energy spread corresponding to the stochastic energy spread (correlations have been removed).

downstream of the rf-gun. The presently achieved parameters (see Table 4) are close to the required LCLS parameters for the low charge operation scenario.

In Japan, Sumitomo Heavy Industry (SHI) has performed beam measurements using a BNL/SLAC/UCLA type rf-gun coupled to an S-band linac ${ }^{47}$. Downstream of the linac, at energies of $14 \mathrm{MeV}$, the transverse emittance dependence on the bunch charge and drive-laser pulse shape and length have been investigated. For a uniform drive-laser time profile, the measured transverse emittance of $1.2 \mathrm{~mm}$-mrad for a charge of $Q=1 n C$ has been reported. The emittance has been measured using the quadrupole scan technique and Gaussian distributions were fitted to the profile in order to extract rms values of the beam size and to infer the transverse emittance.

At CERN, the CLIC linear collider test facility 2 (CTF-2) was constructed at CERN to study the generation of $30 \mathrm{GHz}$ rf (required for the CLIC drive beam). It has been driven by a $3 \mathrm{GHz} 1.5$ cell rf-gun capable of producing the very high charge $(100 \mathrm{nC})$ required for the drive beam. The electron beam requirements are essentially on the peak current, and up to $2.5 \mathrm{kA}$ peak current were obtained after compression. The main parameters of the rf-gun are gathered in Table 4. The CTF-2 facility has also served as a test stand for life-time and quantum efficency studies of Alkali photocathodes ${ }^{48}$.

\section{$4.3 \quad L$-band}

An L-band gun operating at $1.3 \mathrm{GHz}$ has been in use at LANL in the context of the Advanced FEL (AFEL) accelerator. The rf-gun consist of a 10-1/2 cell cavity. This gun alone has driven a far-infrared SASE FEL ${ }^{49}$. The slice emittance has been measured by performing streak camera measurements performed together with a quadrupole scan ${ }^{50}$. Thus the analysis of the beam size evolution versus the 
quadrupole strength for different time slices allows an indirect measurement of the slice emittance (measured slice emittance at $1 \mathrm{nC}$ is about $1.6 \mathrm{~mm}$-mrad). This rf-gun has demonstrated operation with $400 \mathrm{~mA}$ average current per macropulse. The gun design incorporates a Nitrogen vessel to support possible cryogenic operation thereby cutting ohmic losses in half.

The TESLA test facility (TTF) and the Fermilab/Nicadd photoinjector laboratory (FNPL) have both been operated with L-band ( $f=1.3 \mathrm{GHz}$ ) rf-guns of the same design. This 1.6 cell rf-cavity was designed and manufactured at Fermilab ${ }^{51}$. The rf-gun was originally designed in the framework of the TESLA linear collider project to produce long macropulse trains and experimentally its operation with rf-macropulses of $800 \mu \mathrm{sec}$ length was demonstrated. Typical transverse emittances measured in the $17 \mathrm{MeV}$ section of the TTF injector are about $3.5 \mathrm{~mm}$-mrad for a $1 \mathrm{nC}$ bunch ${ }^{52}$. This $\mathrm{rf-gun}$ has driven a vacuum ultraviolet SASE-FEL in the saturation regime ${ }^{53}$. At FNPL, a series of measurements along with their comparison with numerical simulations have been performed on the gun ${ }^{61}$. Recently by immersing the photocathode in a magnetic field and using a linear transformation that decomposed the angular momentum into its transverse momentum components ${ }^{62}$, flat beams (that is beams with $\tilde{\varepsilon}_{y} / \tilde{\varepsilon}_{x} \ll 1$ ) were produced ${ }^{63}$. As far as the TTF facility is concerned, a second generation gun that incorporate a co-axial input coupler is under commissioning at the injector facility of DESY-Zeuthen. The inclusion of a coaxial coupler with a doorknob transition renders the cavity axi-symmetric thereby avoiding the potential transverse emittance degradation due to the time-dependent kick induced by side coupler located at the full-cell as in the present design. This improved rf-gun is foreseen to be incorporated in the TTF-FEL user facility.

At Argonne, the AWA team uses a $1.3 \mathrm{GHz}$ rf-gun to produce very high charge $(\sim 100 \mathrm{nC})$ bunches for beam-driven wakefield acceleration. Similarly to the case aforementioned of CTF-2 (previous section), the figure-of-merit for beam driven plasma acceleration is the peak current of the drive-beam rather than transverse emittances.

\subsection{VHF and UHF band}

In the 90's two low frequency guns have been operated at BOEING and CEABruyère le chatel (France).

At Buyères-le-châtel, the ELSA facility incorporates a $144 \mathrm{MHz}$ rf-gun followed by a linac section operating at $433 \mathrm{MHz}$. Because of the long bunch length (and thus very small linear charge-density) the achieved emittance at $1 \mathrm{nC}$ is about $1 \mathrm{~mm}$-mrad ${ }^{55}$. The ELSA accelerator has been operated with a macrobunch of $150 \mu \mathrm{sec}$ at a frequency of $10 \mathrm{~Hz}$, each macropulse consists of about 2000 bunches. The facility was designed to drive a high power infrared FEL and is about to be upgraded to an X-ray Thomson source ${ }^{22}$.

piot'gunrev: submitted to World Scientific on October 23, 2002 


\begin{tabular}{lrrrrr}
\hline \hline & TTF & FNPL & AWA & AFEL & units \\
\hline bunch charge & $1-8$ & $1-7$ & 100 & $1-4$ & $\mathrm{nC}$ \\
laser pulse length (rms) & 8 & $4-10$ & & 1.5 & $\mathrm{ps}$ \\
peak cathode E-field & 40 & 40 & 80 & 20 & $\mathrm{MV} / \mathrm{m}$ \\
beam energy & 20 & 20 & & $15-20$ & $\mathrm{MeV}$ \\
\hline typical set of data: & 1 & 1 & - & 1 & $\mathrm{nC}$ \\
bunch charge & 17 & 18 & - & 15 & $\mathrm{MeV}$ \\
energy & $0.03(\mathrm{u})$ & 0.25 & - & 0.2 & $\%$ \\
energy spread (rms) & 3.5 & 3.5 & - & 1.6 & $\mathrm{~mm}-\mathrm{mrad}$ \\
$\tilde{\varepsilon}_{\perp}(\mathrm{rms})$ & 8 & 8 & - & 3.5 & $\mathrm{ps}$ \\
bunch length (rms) & & & & &
\end{tabular}

Table 5. Typical photocathode drive-laser and rf parameters for various L-band guns. A set of achieved beam parameters is also given. All the gun presented in this Table operate at $f=1.3 \mathrm{GHz}$. Note: "(u)" in the "energy spread" line indicates the quoted energy spread corresponding to the stochastic energy spread (correlations have been removed).

\begin{tabular}{lrrr}
\hline \hline & ELSA & BOEING & units \\
\hline bunch charge & $1-10$ & $1-7$ & $\mathrm{nC}$ \\
laser pulse length (rms) & 26 & 10 & $\mathrm{ps}$ \\
peak cathode E-field & 25 & 25 & $\mathrm{MV} / \mathrm{m}$ \\
beam energy & 19 & 5 & $\mathrm{MeV}$ \\
\hline typical set of data: & 1 & 3 & $\mathrm{nC}$ \\
bunch charge & 19 & 5 & $\mathrm{MeV}$ \\
energy & $0.1(\mathrm{u})$ & 2 & $\%$ \\
energy spread (rms) & 1 & 5 & $\mathrm{~mm}-\mathrm{mrad}$ \\
$\tilde{\varepsilon}_{\perp}(\mathrm{rms})$ & 25 & 11 & $\mathrm{ps}$ \\
bunch length (rms) & & & \\
\hline \hline
\end{tabular}

Table 6. Typical photocathode drive-laser and rf parameters for the two low frequency guns (ELSA and BOEING). A set of achieved beam parameters is also given. Note: "(u)" in the "energy spread" line indicates the quoted energy spread corresponding to the stochastic energy spread (correlations have been removed).

At BOEING defense and space center, a high average FEL including an energy recovery scheme was operated in the 90's. Noteworthy was the ability to produce electron bunches out of a $433 \mathrm{MHz}$ rf-gun with the record duty cycle of $25 \%$ resulting in an electron beam average power of $160 \mathrm{~kW}^{56}$.

These two low frequency designs operate with rather low electric peak field on the photocathode, about $25 \mathrm{MV} / \mathrm{m}$, and with long (Gaussian) drive-laser pulse length of about $20 \mathrm{ps}$ rms. Because of the latter fact, the charge density is reduced and so are the space charge forces.

piot'gunrev: submitted to World Scientific on October 23, 2002 


\subsection{Superconducting radio-frequency guns}

The operation of a photocathode inside a superconducting resonator was proposed in Reference ${ }^{58}$ and a first test was performed in the early 90 's using a $2.8 \mathrm{GHz}$ niobium cavity ${ }^{59}$. At that time, the cavity peak electric field was only about 1.5 MV/m. Recently, a major breakthrough was achieved by the DROSSEL collaboration: At Forschungzentrum Rossendorf, a superconducting rf-gun ${ }^{57}$ was operated with a field as high as $15 \mathrm{MV} / \mathrm{m}$. The proof-of-principle cavity consists of an half cell of the TESLA cavity geometry with its large diameter side terminated by a shallow cone (see Fig. 3 ) that provides some radial focusing to compensate for the absence of a solenoid ${ }^{a}$ in the vicinity of the cavity ${ }^{23}$. Since the photocathode cannot be in thermal contact with the cavity, a special rf-filter (so-called "choke filter") was implemented to avoid the rf-power from flowing through the cathode coaxial manipulator system thereby preserving the high quality factor of the cavity. At a later stage, the DROSSEL collaboration envisiones to develop a multi-cell cavity superconducting rf-gun to serve as an injector for the ELBE ${ }^{60}$ FEL.



Figure 3. Picture of the Nb half cell cavity for the proof-of-principle srf-gun of Forschungzentrum Rossendorf (Courtesy of D. Janssen).

\section{Conclusion \& Outlook}

Electron sources based on rf-guns have provided electron beams with the required high phase space density to drive short-wavelength single-pass FELs: recently

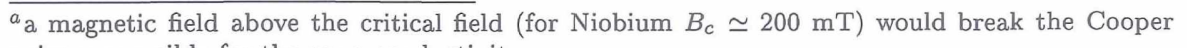
pairs responsible for the superconductivity.

piot'gunrev: submitted to World Scientific on October 23, 2002 
two of these devices were operated in the saturation regime at ANL (LEUTL) and DESY (TTF). Rf-guns can sustain high peak field but cannot simultaneously provide long rf-macropulses: to date the highest duty cycle rf-gun was operated at BOEING with a peak electric field of only $25 \mathrm{MV} / \mathrm{m}$, an electric field comparable to the foreseen achievable field in dc-guns. The latter type of gun having the advantage to be able to drive accelerators in CW mode as demonstrated in the operation of a kW-level average power FEL at JLab.

As far as the choice of the rf-gun frequency is concerned, Figure 4 compares the brightness of the various sources aforementioned. Given the peak current $\hat{I}$, the brightness defined as:

$$
B_{\perp}=\frac{\hat{I}}{\tilde{\varepsilon}_{x} \tilde{\varepsilon}_{y}}
$$

has been compared since the longitudinal emittance (especially the uncorrelated energy spread) has not been measured in all the quoted experiments. In this figure there is a weak frequency dependence of the brightness. Though the gun with a significantly higher frequency (MIT gun) does not yet take advantage of the emittance compensation process. In the latter Figure, the brightness dependence on frequency is mainly dominated by the scaling of the peak current versus frequency ${ }^{b}$ which is in any case increased by compressing the bunch.

The success at FNAL to generate flat beams directly out of the FNPL injector, if included in the design of a future linear collider, would relax the requirements on the electron damping ring. The round-to-flat beam transformation has also found applications in light sources ${ }^{64,65}$. If one would be able to generate polarized flat beams directly out of an injector, one might be able to remove the need for an electron damping ring. The topic of rf-gun for producing polarized electrons is under investigation ${ }^{66,67}$, the main challenge being to operate GaAs photocathodes in an rf-gun (or finding another photo-emitter capable of producing polarized electrons). It was suggested that the plane wave transformer rf structure may be a good candidate for such an polarized rf-gun since it has a larger vacuum conductance (compared to standard gun cavities) and thereby offers a higher quality vacuum environment ${ }^{68}$.

\section{Acknowledgments}

I wish to thanks the following individuals for providing materials: J.-P. Carneiro (DESY), D. Janssen (Forschungzentrum Rossendorf), V. Yakimenko F. Zhou (BNL/ATF), W. Graves (BNL/SDL), C. Sinclair (Cornell), Ph. Guimbal (CEA Buyères-le-châtel), R. Temkin (MIT), D. Dowell, J. Schmerge (SLAC/GTF), M. Useaka (Univ. Tokio), and J. Yang (Sumitomo Heavy Industry Ltd.) and K. Flöttmann for discussions. I am also indebted to J.-P. Carneiro and K. Flöttmann for reading and commenting the manuscript.

${ }^{b}$ typically we have $\hat{I} \propto \omega$ for the presently operated guns. 




Figure 4. Comparison of the brightness of various sources quoted in the paper. In this plot the brightness has been computed using Eq.(6).

\section{References}

1. Sheffield R., proc. PAC1995 Dallas, 882-886 (1996)

2. O'Shea P., "Rf photoinjectors", proceedings of 1st ICFA workshop on "high brightness beam" UCLA, published by World Scientific (1999)

3. Carsten B., Nucl. Instr. Meth. A285:313-319 (1989)

4. Carsten B., Part. Acc. 49:27-65 (1995)

5. Serafini L. and Rosenzweig J., Phys. Rev. E55:7565-7590 (1997)

6. Piot P. et al, proceedings EPAC1998 Stockholm, 1447-1449 (1998)

7. Wang X.J., et al. Nucl. Instr. Meth. A375:82-86 (1996)

8. Travish G., et al., proc. LINAC2000 Monterey (report SLAC-R-561), 899-901 (2000)

9. Yu. L.-H., et al., proc. PAC2001 Chicago, 2830-2832 (2001)

10. Braun H. H., et al., proc. PAC2001 Chicago, 720-722 (2001)

11. Sheffied R., et al., Nucl. Instr. Meth A318:313-319 (1989)

12. Conde M. et al., Phys. Rev. ST Accel. Beams 1:041302 (1998)

13. Schmerge J. et al., Nucl. Instr. Meth A483:301-304 (2002)

14. Schreiber S., proc. EPAC2000 Wien, 86-88 (2000)

15. Bakker R., et al, proc. EPAC2002 Paris, 1813-1815 (2002)

16. Carneiro J.-P., et al, proc. PAC1999 New-York, 2027-2029 (1999)

17. Brown W. J., et al, Phys. Rev. ST Accel. Beams 4:083501 (2001)

18. Uesaka M. et al., IEEE trans. plasma science 28(4): 1133-1142 (2000)

19. Anderson S., et al., proc. PAC1999 New-York, 2006-2008 (1999) 
20. Teifer S., et al., proc. PAC2001 Chicago, 2263-2265 (2001)

21. Dowell D., et al, proc. PAC1993 Washington, 2967-2969 (1993)

22. Guimbal Ph., et al., proc. EPAC2002 Paris, 1768-1770 (2002)

23. Janssen D.,and Volkov V., Nucl. Instr. Meth. A452:34-43 (2000)

24. Rosenzweig J.B., et al., proc. PAC1999 New-York, 2039-2041 (1999)

25. Sinclair C.K., proc. PAC1999 New-York, 65-69 (1999)

26. Sinclair C.K., ICFA beam dynamics newsletter 26:31-34 (December 2001)

27. Kim K.J., Zholents A.A, Zolotorev M.S., Vinokurov N.A., Nucl. Instr. Meth. A407:380-384 (1998)

28. Faatz B. et al., "VUV-FEL driven rf-gun", proc. FEL2002 Argonne (in press)

29. Spicer W.E., Herrera-Cómez M., "Modern theory and applications of photocathodes", report SLAC-PUB-63-06 and SLAC/SSRF-0042 (1993)

30. Flöttmann K., DESY-HH report TESLA-97-06 (1997)

31. Dunham B.M., et al, proc. PAC1995 Dallas, 1030-1032 (1995)

32. Orlov D.A., et al, Appl. Phys. Lett. 78(18):2721-2723 (2001)

33. Graves W., et al, proc. PAC2001 Chicago, 1030-1032 (2001)

34. Wang J.W. and Loew G.A., SLAC-PUB-7684 Stanford Univ. (1997)

35. Engwall D., et al., proc. PAC1997 Vancouver, 909-911 (1998)

36. Neil G.R., et al, Phy. Rev. Lett. 84(4):662-665 (2000)

37. Sinclair C.K., proc. PAC2001 Chicago, 610-612 (2001)

38. Hovater C., and Poekler M., Nucl. Instr. Meth. A418:280-286 (1998)

39. Gruner S., et al. "Study for a proposed phase I ERL synchrotron light source at Cornell university", CHESS TM-01-003 and JLAB-ACT-01-04 (2001)

40. Rosenzweig J. and Colby E., proc. PAC1995 Dallas, 951-953 (1996)

41. Lin L. C.-L., proc. PAC1995 Dallas, 957-959 (1996)

42. Zhou F., et al. Phys. Rev. ST Accel. Beams 5:094203 (2002)

43. Yakimenko V., et al., , Nucl. Instr. Meth A483:277-281 (2002)

44. Graves, W. et al, proc. PAC2001 Chicago, 2224-2226 (2001)

45. Piot $\mathrm{Ph}$. et al., "Sub-picosecond compression by velocity bunching in a photoinjector", this proceedings (also preprint TESLA-FEL-02-08)

46. Dowell D., et al., "Slice Emittance Measurements at the SLAC Gun Test Facility", submitted to proc. FEL2002 Argonne (in press)

47. Yang J., et al., proc. EPAC2002 Paris, 1828-1830 (2002)

48. Suberlucq G., "Développement et production de photocathodes pour la CLIC Test Facility", CLIC Note 299, CERN (1996)

49. Hogan M., et al, Phy. Rev. Lett. 81(22):4867-4870 (1998)

50. Gierman S., "Enhanced quadrupole scan technique for slice emittance measurement", PhD thesis, University of California San Diego (defended in 1999)

51. Colby E. R., et al., proc. PAC1995 Dallas, 967-969 (1995)

52. Piot Ph., et al., proc. PAC2001 Chicago, 86-88 (2001)

53. Ayvazian V., et al., Eur. Phys. Jour. D20:149-155 (2002)

54. Schreiber S., et al., proc. EPAC2002 Paris, 1804-1806 (2002)

55. Marmouget J.-G., et al., proc. EPAC2002 Paris, 1795-1797 (2002)

56. Dowell D., et al., Appl. Phys. Lett. 63(15):2035-2037 (2002)

57. Janssen D., private communication; H. Büttig et al., "First operation results of a superconducting rf-photoelectron gun", submitted to proc. FEL2002 Ar-

piot'gunrev: submitted to World Scientific on October 23, 2002 
gonne (in press)

58. Chaloupka H. et al., Nucl. Instr. Meth. A285:327-332 (1989)

59. Michalke et al., proc. EPAC1992 Berlin, 1014-1116 (1992)

60. Büchner A., et al., proc. EPAC2000 Wien, 732-734 (2000)

61. Carneiro J.-P., "Etude expérimentale du photoinjecteur de Fermilab" thèse de l'Université Paris Sud XI (defended in 2001)

62. Brinkmann R., Derbenev Ya, and Flöttmann K., Phys. Rev. ST Accel. Beams 4:053501 (2001)

63. Edwards D. et al., proc. PAC2001 Chicago, 73-75 (2001)

64. Lydia S., et al., this proceedings

65. Brinkmann R., proc. EPAC2002 Paris, 653-655 (2002)

66. Dikansky N.S., et al., proc. EPAC2000, 1645-1647 (2000)

67. Edwards D. (chairperson), ICFA mini-workshop on "Polarized rf-guns for linear colliders", FNAL April 18-20, 2001

68. Clendemin J., et al., "A polarized electron RF photoinjector using the planewave-transformer design", submitted to Phys. Rev. ST Accel. Beams and preprint SLAC-PUB-8971 (2001) 